\title{
TWO CASES OF CHANGES IN AMINO ACID TRANSPORT ACTIVITY WITH TISSUE DEVELOPMENT
}

\author{
Halvor N. Christensen \\ Department of Biological Chemistry, The University of \\ Michigan, Ann Arbor, Michigan
}

My contribution to this symposium relates to the changes in the amino acid transport activity of two tissues in association with developmental changes. One of these is the abrupt intensification of the degree to which test amino acids are concentrated into the liver, which occurs in the guinea-pig during the first day of postnatal life.(1) The other is the loss of two or more uphill transport systems for such amino acids as glycine and alanine, on the maturation of the rabbit reticulocyte. (2)

The observations of amino acid distribution in the guinea-pig made use of the endogenously present glycine, and in addition an unmetabolizable model amino acid, 1-aminocyclopentanecarboxylic acid, which had been injected 2 or 3 days before the piglets were delivered. Figure 1 shows the structure of the

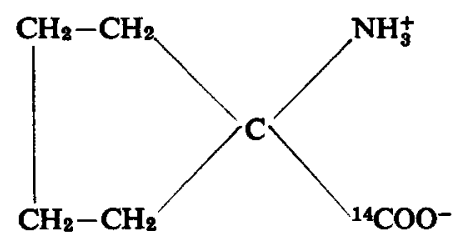

Fro. 1

Structure of 1-aminocyclopentanecarboxylic acid-1-14C ("Cycloleucine").

amino acid used. This amino acid is lost only very slowly from the body because of its excellent renal tubular resorption. (3) At the selected time after delivery, blood was taken from the heart under ether anesthesia, and the liver and the muscles of one thigh removed and extracted by grinding in 10 parts of $0.01 \mathrm{~N}$ acetic acid. The resulting suspensions were held at $100^{\circ}$ for $3 \mathrm{~min}$, and then centrifuged to remove coagulated material.

Deproteinized filtrates of the plasma, and the tissue extracts, were analyzed colorimetrically for glycine by measuring the formaldehyde released by the 
action of ninhydrin, and for 1 -aminocyclopentanecarboxylic acid-1-14 $\mathrm{C}$ by liquid-scintillation counting. In some cases delivery was made by section.

Figure 2 shows that the degree to which "cycloleucine" is concentrated by the liver, with respect to the plasma, rises from a mean value of 0.9 at birth to a value above 2.0 in $24 \mathrm{hr}$. Little further rise occurs in the following days. The behavior is similar for glycine, the distribution ratio rising from 4.4 to about 20 in the same interval. The latter value may be compared with the adult ratio, which averages about 33 .

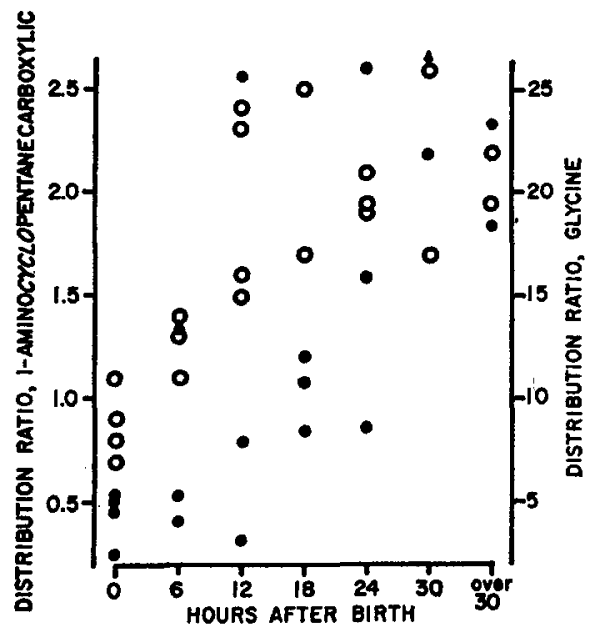

FIG. 2

Change in the ratio of distribution of 1-aminocyclopentanecarboxylic acid (open circles) and of glycine (closed circles) between the liver cell water and the plasma in the guinea-pig after birth. Scale at the left is for "cycloleucine"; the scale at the right, for glycine. Reproduced from J. Biol. Chem.(1) with permission.

Analyses of the muscle sample provide a standard for comparison. As Fig. 3 shows, the degree to which glycine is concentrated does not change during the neonatal interval, the ratio remaining at about 6 or 7 to 1 . In the case of cycloleucine there is an 18 per cent rise in the distribution ratio, a change that we may logically attribute to the rapid decline in the aggregate level of amino acids that compete with cycloleucine for uptake during this interval. That is, before delivery the concentrative activity of the placenta keeps the plasma amino acid level some five times higher than the postnatal level. Under these conditions one would expect some increase after delivery in the steepness of concentration of an amino acid with an appropriately placed $K_{m}$ for transport. In any case the change in the muscle is very small in relation to that shown by the liver. 
The two amino acids employed make complementary contributions to the demonstration: The results with cycloleucine show that the change in the liver level does not arise from one improbable source, namely decreased rate of hepatic utilization, since this amino acid suffers no utilization; the results with glycine show that intensified uptake by the liver is strong enough not to be cancelled out by a probably faster utilization during this time when many new proteins are being synthesized.

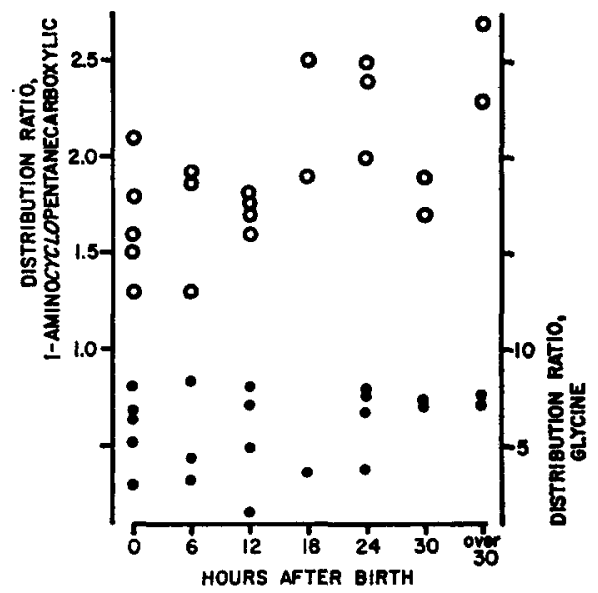

Fia. 3

Changes of distribution of the same amino acids for muscle. The two amino acids are designated just as in Fig. 2. Reproduced from J. Biol. Chem.(1) with permission.

Nemeth and others ${ }^{(4)}$ have shown that the adult metabolic roles of the liver are established during the early postnatal period and that a number of enzymes characteristic of these roles appear during this interval. It is interesting that the transport activity for amino acids is intensified during the same interval.

The observations on red blood cells were made with rabbit cells, because of the ease of producing a reticulocytosis with phenylhydrazine. The red cells were separated by centrifugation, and their uptake of ${ }^{14} \mathrm{C}$-labeled amino acids studied in dilute suspension in the saline medium of Raker et al. at $37^{\circ} .{ }^{(5)}$ Uptake was terminated after a brief measured interval by diluting the cell suspension with ice-cold buffered medium, and the cells separated by 2 min of centrifugation in the cold. Deproteinized extracts of the two phases were then analyzed by liquid-scintillation counting.

Figure 4 shows some new results obtained by Dr. C. G. Winter in my laboratory, which confirm and extend results obtained by Dr. Riggs and myself 
a dozen years ago.(6) The solid lines show observations with the reticulocyterich populations (50-80 per cent reticulocytes); the dashed lines with those for the ordinary blood cell population. The solid round points are for leucine, which already attains a steady-state distribution for both cell-populations in $1 \mathrm{~min}$. Uptake of valine takes somewhat longer, but the results are again the same for both populations. The triangles represent alanine; the squares, glycine. In the case of the latter two amino acids the uptake is much faster in the reticulocyte, and continues until substantial concentration gradients are generated.

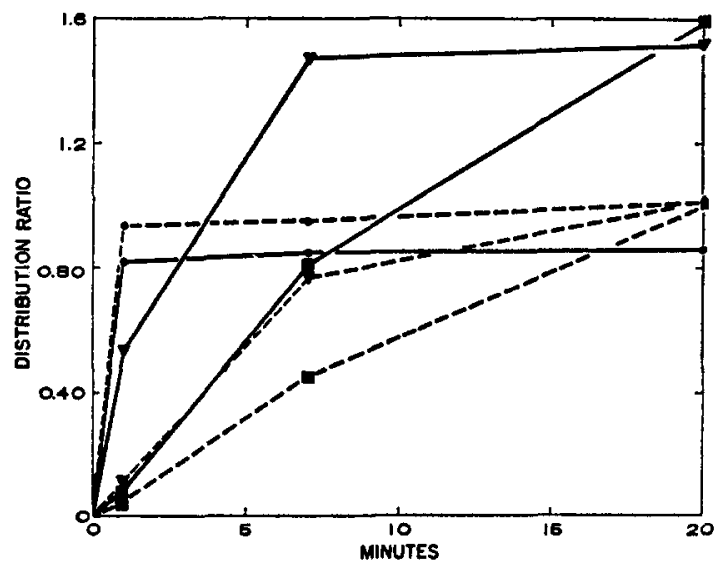

FIG. 4

Course of the uptake of L-leucine, L-alanine and glycine by rabbit erythrocytes and reticulocytes. See text for description. Reproduced from J. Biol. Chem., with permission.

We wished then to classify the transport systems that do and do not survive maturation of the reticulocyte. You will perhaps recall that the action of insulin on amino acid transport is most conspicuous for a small group of amino acids that tend to be transported steeply uphill into tissues.(7) Our preliminary hypothesis was that a system of the leucine-preferring type (as described for the Ehrlich cell)(8) survived and that one of the alanine-preferring type did not survive maturation. This supposition proved correct in the first instance: a rapidly-exchanging, non-concentrative system operates for valine and leucine at both stages. This system has the same sort of insensitivity to $\mathrm{pH}$ and the distribution of the alkali-metal ions that we see for the leucinepreferring system in the Ehrlich cell.

The non-surviving transport activity has 3 features seen for the alaninepreferring system in the Ehrlich cell:(8) It is more sensitive to lowering of the $\mathrm{pH}$; it is sensitive to the alkali metal distribution; and it operates uphill. But, as Table 1 shows, measurement of the kinetic constants fails to identify 
TABLE 1

$K_{m}$ and $K_{i}$ Values for the Uptake of Four Amino Acids by the Rabbit Erythrocyte and Reticulocyte

Values in parentheses are mean $K_{m}$ values; the other numbers are $K_{t}$ values obtained by the method of Dixon. Bracketed values are from replicate experiments

\begin{tabular}{l|c|c|c|c|c}
\hline \multirow{2}{*}{ Cell type } & \multirow{2}{*}{$\begin{array}{c}\text { By inhibition of } \\
\text { uptake of }\end{array}$} & \multicolumn{4}{|c}{ Constants estimated for } \\
\cline { 3 - 6 } & L-leucine & L-valine & L-alanine & glycine \\
\hline Erythrocyte & L-leucine & $(1.1)$ & 2.6 & $>200$ & $>200$ \\
& L-valine & $\left\{\begin{array}{l}1.1 \\
0.62\end{array}\right.$ & $(2.8)$ & $>200$ & $>200$ \\
\hline Reticulocyte & L-valine & 1.2 & $(2.8)$ & $\gg 2$ & $\gg 40$ \\
& L-alanine & $\left\{\begin{array}{l}1.4 \\
0.92\end{array}\right.$ & $\left\{\begin{array}{l}2.5 \\
2.3\end{array}\right.$ & $(0.22)$ & $\left\{\begin{array}{l}4.7 \\
3.9\end{array}\right.$ \\
& glycine I & $\left\{\begin{array}{l}5.3 \\
4.5\end{array}\right.$ & $\left\{\begin{array}{l}11 \\
9.1\end{array}\right.$ & $\left\{\begin{array}{c}0.92 \\
0.34\end{array}\right.$ & $(6.1)$ \\
& glycine II & 28 & 52 & 9.7 & $(0.039)$ \\
\hline
\end{tabular}

it as a simple, homogeneous system. One sees here $K_{m}$ and $K_{i}$ values obtained by applying the Michaelis-Menten model to initial-rate values for uptake. Uptake intervals used ranged from min to 3 min. It may be seen that the constants describing the inhibitory actions of leucine, valine and alanine on the uptake of alanine are in each case about 4 times as large as those applying to the main mode of glycine uptake. Furthermore, the transport of alanine in the reticulocyte has a strong countertransport activity, whereas that for glycine failed to show any. Therefore we conclude that we are dealing with at least two different transport systems still present at the reticulocyte stage. To add to the complexity, note that we have listed two sets of constants for glycine transport: the second one, listed as glycine II, appears to have a considerable preference for glycine over other amino acids. This second, high-affinity route could well correspond to a specific glycine transport of the pigeon red blood cell which Vidaver at this University has shown recently to be closely coupled to the down-gradient migration of $\mathrm{Na}^{+}$. Figure 5 shows the basis for introducing these subclassifications for glycine transport. The Lineweaver-Burk plot corresponds rather well with the presence of two modes of glycine transport.

However we interpret this behavior, the preceding table and figure reveal enough complexity to force us to abandon the view that only one amino acid transport system is lost on maturation of the reticulocyte. A more interesting question than the exact categorization of the several systems lost is why they are lost. Does uphill transport disappear because the level of ATP generated is no longer high enough to support these systems? We should 
be able to answer that question by seeing what happens when ATP is introduced into erythrocyte ghosts. Or are these uphill transport activities lost as a necessary consequence of the loss of protein synthesis?

You will recall that Borsook and his associates ${ }^{(9)}$ showed that ability of the reticulocyte to incorporate leucine into globin is lost on maturation of the reticulocyte. The present results illustrate again the general tendency of high rates of protein synthesis to be associated with heightened activity for amino

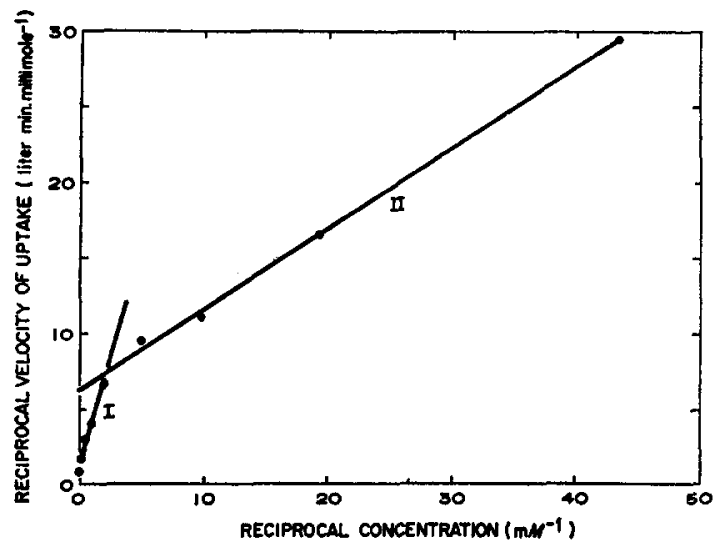

FIG. 5

Lineweaver-Burk plot of glycine uptake by reticulocyte-rich red cell populations. The values for the kinetic constants describing the two lines (see Table 1) were obtained by successive approximation. Reproduced from J. Biol. Chem.(2) with permission.

acid transport (cf. 10). As Dr. Weber showed us yesterday, the elevation of amino acid levels occurs as early as any hepatic effect of glucocorticoid administration. And you will recall that this action occurs for amino acids that cannot possibly have been produced by acceleration of transamination or any other enzymatic reaction.(11) We can now perhaps safely conclude, as illustrated in Fig. 6, that neither of the two effects, namely, the intensification of amino acid uptake and the acceleration of protein synthesis is necessary, one for the other, although they can scarcely avoid influencing one another. Instead primary actions on growth and protein synthesis must be of a nature to cause both effects. I will cite two pieces of evidence for this view, both related to hormone actions on the isolated rat diaphragm. An experiment by Kostyo shows that growth hormone can stimulate incorporation of amino acids into the protein of the diaphragm even when its action to stimulate amino acid entry into the tissue is prevented by the absence of sodium ion.(12) Conversely, Fritz and Knobil showed that the insulin stimulation of protein 
synthesis in the diaphragm can be prevented by the presence of puromycin, and yet the action of the hormone on amino acid uptake is preserved.(13)

Accordingly, the two events, the stimulation of amino acid transport and the stimulation of protein synthesis appear to lie on a branched chain of events, rather than lying in sequence in a single cause-and-effect chain. The position of the stimulation by hormones of nucleic acid synthesis on such chains apparently remains to be demonstrated.

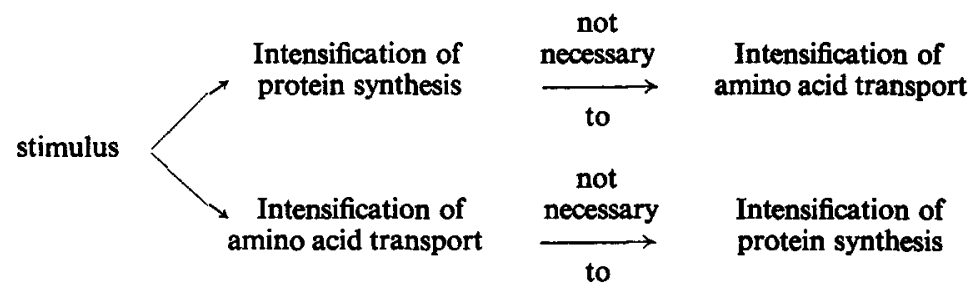

FIG. 6

Question of the relation between two consequences of the stimulation of protein synthesis.

\section{SUMMARY}

During the first day of extrauterine life the liver of the guinea-pig greatly intensifies the extent of the steady-state concentration of two amino acids, one normal and one non-metabolizäle. Another change with tissue development is in the reverse direction, namely the loss of two or more uphill transport systems by the rabbit reticulocyte on its maturation. The nature of the association between changes in the character and intensity of protein synthesis and in amino acid transport activity, as illustrated by these two cases, is discussed.

\section{ACKNOWLEDGMENT}

I am of course speaking for the several persons who have actually performed the experiments described, as cited below. The work from my laboratory was partially supported by a grant (CA-02645) from the National Cancer Institute, National Institutes of Health, U.S. Public Health Service.

\section{REFERENCES}

1. H. N. Christensen and J. B. Clifford, Early postnatal intensification of hepatic accumulation of amino acids, $J$. Biol. Chem. 238, 1743-1745 (1963).

2. C. G. Winter and H. N. Christensen, Contrasts in neutral amino acid transport by rabbit erythrocytes and reticulocytes, Manuscript in preparation.

3. H. N. ChrISTENSEN and J. C. JoNes, Amino acid transport models: Renal resorption and resistance to metabolic attack, J. Biol. Chem. 237, 1203-1206 (1962). 
4. A. M. NEMETH, Glucose-6-phosphatase in the liver of the fetal guinea pig, J. Biol. Chem. 208, 773-776 (1954).

5. J. W. RAKer, I. M. TAYLOR, J. M. Weller and A. B. HAstings, Rate of potassium exchange of the human erythrocyte, J. Gen. Physiol. 33, 691-702 (1950).

6. T. R. Rigas, H. N. Christensen and I. M. Palatine, Concentrating activity of reticulocytes for glycine, J. Biol. Chem. 194, 53-55 (1952).

7. H. N. Chrustensen, Biological Transport, pp. 58, New York (1962).

8. D. L. OXENDER and H. N. Christensen, Distinct mediating systems for the transport of neutral amino acids by the Ehrlich cell, J. Biol. Chem. 238, 3686-3699 (1963).

9. H. Borsook, C. L. Deasy, A. J. HaAgen-Smith, G. Keighiley and P. H. Lowy, In vitro incorporation of labeled leucine into the proteins of rabbit reticulocytes, Federation Proc. 10, 18 (1951).

10. H. N. Christensen, Mode of transport of amino acids into cells, pp. 63-106 in Amino Acid Metabolism (W. D. McElroY and B. Glass, eds.), Baltimore (1955).

11. M. W. Noall, T. R. Riggs, L. M. WalkeR and H. N. Christensen, Endocrine control of amino acid transfer, Science 126, 1002-1005 (1957).

12. J. L. KostYo, Separation of the effects of growth hormone on muscle amino acid transport and protein synthesis, Endocrinology 75, 113-119 (1964).

13. G. R. FrITZ and E. KNOBIL, Amino acid transport in the absence of protein synthesis: Stimulation by insulin, Federation Proc. 23, 409 (1964). 